\title{
LESSON 19
}

The Tabulator

Warm up

MARGINS:

Left, Pica 20; Elite 30;

Right, moved out of the way.

PAPER: Against the paper guide at 0 on the scale on the left. Turn up 7 single lines.

The question mark (?)

Leave 2 spaces after a question mark.

The shift lock

Spacing after punctuation
All standard typewriters have a tabulator mechanism which enables the typist to find any given point or points very quickly and easily. The method of operation varies slightly from machine to machine and your teacher will tell you how your machine works.

As a general rule, the tabulator bar is found at the top of the keyboard and you should press it down with the $\mathrm{j}$ or $\mathrm{f}$ finger until it stops moving.

1. To clear a tab key press the key marked CLEAR or - (minus).

2. To set a tab key press the key marked SET or + (plus) at the desired point.

Clear all existing tabs, after you have moved both margins out of the way, and practise setting tab keys by setting them at 10, 20, 40,60 and 70 .

The tabulator will help you when you type indented paragraphs (Lesson 16) and hanging paragraphs (Lesson 18).

Use the indented paragraph style and type this alphabet review paragraph as many times as your teacher asks.

Five jewel cases by the gate were quite heavy and the mules pulled extra hard to keep going over the frozen ground.

The position of this key varies from machine to machine and your teacher will give you the first line of the drill. Type each line 3 times.

1. What? May I? How many? So? What for? My turn?

2. Will she come? At what time? And? Where is Ray?

3. Only fl5 father? Why? Why not $f 9$ at least? How?

When typing several capital letters it is easier to use the shift lock than to use the shift key for each letter. Move the a finger to the left and press down the shift lock key while you type the required capitals and then use the shift key to release the lock. On some machines, either shift key will release the lock but on others only the left one will. Type each line 3 times.

4. THIS is the FIRST TIME I have used the SHIFT LOCK.

5. It WILL NOT be the LAST TIME that I shall need it.

6. This train will call at STAFFORD, CREWE and DERBY.

There are several recognized rules about spacing after punctuation. The main thing is to be consistent. This book uses the following methods:

1. Leave 2 spaces after a full stop at the end of a sentence, a question mark or an exclamation mark.

2. Leave one space after a comma, colon or semi-colon.

3. Leave one space after a person's initials, and the abbreviations Mr. and Mrs. and Messrs., e.g., Mr. M. T. Roberts, Mrs. B. A. Rice.

4. No space is left after some abbreviations, e.g., C.O.D.B.Sc. p.m. f.o.b. Between groups of degrees leave a space, e.g., B.A., B.SC. 